\title{
LES DIMENSIONS COLLECTIVES DE L'ACTUALITÉ LOCALE : VERS UNE GRILLE D'ANALYSE DES PRATIQUES DES ACTEURS DES ÉCOSYSTÈMES MÉDIATIQUES
}

\author{
Victor WIARD $^{1}$
}

S'insérant dans le débat académique portant sur les pratiques de participation au journalisme et le journalisme participatif, cet article propose une grille d'analyse des pratiques des acteurs impliqués dans la production de nouvelles locales sur Internet. Au moyen d'une revue des différents modèles existants dans la littérature, nous proposons le modèle «ADD élargi » pour accès, dialogue, délibération. En outre, nous proposons de croiser ces trois aspects de la participation au journalisme la diversité des catégories d'acteurs impliqués - les journalistes professionnels, mais aussi les politiciens et activistes locaux ainsi que d'autres citoyens - afin de mieux comprendre les interactions entre les différents acteurs des écosystèmes médiatiques locaux. In fine, cette approche propose un autre regard sur la relation entre les individus qui interviennent d'une manière ou d'une autre dans la production de nouvelles locales.

\section{Introduction}

$\mathrm{Au}$ sein des sciences de l'information et de la communication, les recherches portant sur le journalisme et les médias se sont approprié le

1 Christophe WIARD est chercheur à l'Université Saint-Louis Bruxelles au sein du laboratoire « Engage - Research Center for Publicness in Contemporary Communication » (Belgique).

Recherches en communication, $\mathrm{n}^{\circ} 52$ - Article publié le 08/09/2021 
concept de participation, dans le but de mettre en évidence les mécanismes qui lient les citoyens aux journalistes professionnels au cours de la production journalistique. Ainsi, le terme de « journalisme participatif » fait référence à des pratiques diverses, qui impliquent d'une façon ou d'une autre le rôle actif du public dans la production d'informations liées à l'actualité (Carpentier, 2016 ; Paulussen et al., 2007). Dans ce contexte, la communauté scientifique s'est notamment intéressée, ces 20 dernières années, au potentiel démocratique du journalisme participatif (Borger et al., 2013). De ce point de vue, le terme prend une connotation positive : il est alors vu comme créateur de lien entre journalistes et citoyens ou comme vecteur de crédibilité pour les institutions journalistiques (Singer et al., 2011). À l'inverse, le journalisme participatif peut également être perçu comme problématique, la participation citoyenne étant parfois perçue comme incontrôlable et non professionnelle, voire intentionnellement néfaste (Quandt, 2018).

Plus récemment, la numérisation de la production de l'information (Kreiss \& Brennen, 2016) a poussé les chercheurs à élargir l'usage du concept de participation pour décrire, au-delà du lien entre citoyens et journalistes professionnels, la diversité des formes que cette participation peut prendre (allant du choix des sujets à la coproduction de contenus, en passant par le partage d'images ou la diffusion sur les réseaux sociaux). Dans ce contexte, plusieurs études ont également analysé la manière dont ces formes de participation peuvent varier selon les acteurs impliqués (citoyens, activistes, journalistes amateurs, politiciens, etc.). Pourtant, les interactions entre ces acteurs sont multiples et complexes. Il apparaît dès lors pertinent de mettre en place une méthode qui soit ouverte à cette diversité, une grille d'analyse qui permette de mettre ces pratiques et acteurs en perspective. Après une revue de la littérature relative aux écosystèmes médiatiques locaux et à la participation au journalisme, nous comparerons les différents modèles utilisés pour analyser ces pratiques (Domingo et al., 2008 ; Carpentier, 2011, 2016 ; Kammer, 2013 ; Ahva, 2017 ; Ahva \& Wiard, 2018). Nous proposerons ensuite le nôtre. Il s'agira, en substance, d'un tableau croisé des acteurs potentiels et des pratiques participatives divisées en trois catégories : l'accès au processus de production de contenus, le dialogue durant celui-ci et la délibération sur sa forme finale. 


\section{Cadre conceptuel}

\subsection{La reconfiguration des pratiques journalistiques dans les écosystèmes médiatiques locaux au $\mathrm{XXI}^{e}$ siècle}

Nombre de chercheurs se sont penchés sur les caractéristiques spécifiques de la production journalistique dans le contexte local (voir à ce sujet, dans le contexte anglo-saxon, Franklin, 2005 ; Aldrige, 2007 ; Wall, 2015, et dans le contexte francophone, Bousquet \& Smyrnaios, 2012). Tout comme aux autres niveaux (national et international), le journalisme local a connu, au cours des dernières décennies, des reconfigurations dues en partie à la généralisation de l'usage d'Internet et des réseaux sociaux. Bowman \& Willis affirment, par exemple, qu'un «nouvel écosystème médiatique » est apparu, qui rend le journalisme plus participatif et qui donne la possibilité au public non seulement de consommer, mais aussi de commenter et produire du contenu (Bowman \& Willis, 2003). Ces évolutions technologiques ont provoqué des changements majeurs dans la façon dont les contenus liés à l'information locale sont produits (liens entre public et journalistes locaux en ligne, déclin de l'industrie médiatique locale, etc.). Néanmoins, l'objectif n'est pas ici de dresser une liste exhaustive de ces changements. Il semble toutefois que la métaphore de «l'écosystème » permet d'en comprendre certains ressorts, tant elle permet de penser la complexité des environnements technologiques et sociaux dans lesquels les contenus sont produits (Wahl-Jorgensen, 2016, p. 15). Anderson définit les écosystèmes médiatiques comme :

«l'ensemble des individus, organisations et technologies au sein d'une communauté géographique particulière ou d'un problème spécifique, engagés dans la production et la consommation journalistique » (Anderson, 2016, p. 412).

Un premier volet de la recherche dans ce domaine s'est concentré sur les écosystèmes en ligne, dans lesquels des sites webs sont liés les unes aux autres par le biais de liens hypertextes. Dans les années 2000, le blogging est devenu partie intégrante des pratiques journalistiques (Le Cam, 2006 ; Pélissier \& Diouma Diallo, 2015). Ces sites ont constitué un domaine d'étude particulièrement riche en raison de la popularisation des blogs d'actualité (professionnels ou non) et du développement des possibilités techniques permettant d'analyser les liens entre eux. Si les 
blogs ont, tout d'abord, été considérés comme une menace pour le journalisme professionnel, la recherche montre que, quelles que soient la plateforme utilisée et la diversité des acteurs en présence, les médias traditionnels jouent toujours un rôle central. Plusieurs chercheurs ont également affirmé que les blogs et les sites d'information traditionnels entretiennent une relation plus complémentaire que compétitive (Reese et al., 2007). Au-delà de la question des blogs d'actualité, les écosystèmes des sites d'information présentent également des spécificités qui leur sont propres.

Au niveau local, à Bruxelles, ce courant de recherche montre que les contenus liés à l'actualité produits dans ces écosystèmes sont disséminés par un réseau d'acteurs interconnectés, comprenant des journalistes professionnels travaillant pour des médias privés et publics (locaux et nationaux), des blogueurs amateurs et activistes, ainsi que des sources institutionnelles (Domingo \& Le Cam, 2014; Wiard \& Pereira, 2018). Ryfe et al. ont utilisé une approche similaire pour étudier l'écosystème des sites web de la région de la baie de San Francisco. Ils ont constaté que, si la capacité d'agenda-setting des sites web de médias traditionnels est aujourd'hui affaiblie, ce n'est pas tant lié aux caractéristiques de l'écosystème (par exemple, au fait qu'il y aurait trop de médias d'information) qu'à leurs propres pratiques. Ryfe et al. affirment que les entreprises médiatiques locales analysées semblent réticentes à employer des liens hypertextes qui redirigeraient vers des sources non professionnelles, et qu'elles préfèrent les liens internes - vers d'autres pages de leurs propres sites - en raison d'impératifs commerciaux (Ryfe et al., 2012).

La notion d'écosystème médiatique local a également été réfléchie dans une deuxième perspective de recherche qui, au-delà d'Internet, prend en considération la diversité des espaces de communication dans lesquels circulent les informations. Un groupe restreint, mais productif, de chercheurs a utilisé cette notion pour analyser la production, la circulation et les utilisations des contenus liés à l'actualité en tentant d'évaluer la diversité des processus, supports et acteurs potentiellement impliqués (Domingo, Masip \& Costera-Meijer, 2015). Ces chercheurs ont utilisé la notion d'écosystème pour penser les dimensions collectives de l'actualité en tant que résultat de processus complexes impliquant des acteurs divers, en différents lieux et via divers médias. Ces écosystèmes ont principalement été étudiés aux États-Unis (Anderson, 2010, 2013, 2016), au Royaume-Uni (Anderson, Coleman \& Thumim, 2015 ; Coleman et al., 2016), en France (Powers, Zambrano \& Baisnée, 
2015) et en Belgique (Le Cam \& Domingo, 2015 ; Wiard \& Pereira, 2018).

Dans son étude de 2012, Lowrey s'est concentré sur deux villes américaines et a comparé l'évolution de leurs écosystèmes médiatiques, en interrogeant des producteurs de médias traditionnels ${ }^{2}$ ainsi que des producteurs «alternatifs» (citoyens, blogueurs semi-professionnels, activistes locaux, etc.). Lowrey a découvert que les médias émergents et alternatifs en ligne développent des pratiques de mimétisme vis-à-vis des médias traditionnels, bien qu'ils essaient de trouver des niches non couvertes par ceux-ci. Ces niches peuvent être des zones géographiques (quartiers généralement ignorés), un groupe démographique spécifique (par exemple, une minorité ethnique) ou des thèmes tels que l'art et les loisirs (Lowrey, 2012, p. 271). Napoli et al. ont utilisé le même type d'approche pour évaluer l'état des « écosystèmes journalistiques locaux » dans trois communautés du New Jersey : Newark, New Brunswick et Morristown. Leur objectif était de quantifier l'infrastructure et les performances des producteurs de contenus liés à l'actualité dans ces trois communautés. Ils ont constaté des disparités importantes entre les trois villes, notamment le fait que la quantité et la qualité des contenus produits (au prorata du nombre d'habitants) varient, ce qui montre que les citoyens ne sont pas égaux dans les écosystèmes médiatiques locaux (Napoli et al., 2015). Ces conclusions ont été confirmées par Powers, Zambrano \& Baisnée, qui ont comparé les nouveaux écosystèmes de Toulouse (France) et de Seattle (États-Unis). Même si les deux écosystèmes ont été perturbés par le passage au numérique et les changements technologiques et économiques associés, Toulouse a été moins impactée du fait d'aides publiques et de l'introduction de quotidiens gratuits dans la ville (Powers, Zambrano \& Baisnée, 2015). Finalement, la recherche la plus complète à ce jour sur un écosystème délimité géographiquement a été réalisée par Coleman et al. (2016) qui ont décrit l'écosystème médiatique de Leeds. Leurs résultats montrent que : (1) il y a une forte demande en matière d'informations locales de la part des citoyens, (2) même avec Internet, le public dépend

2 Dans cet article, nous utilisons les termes « médias traditionnels » et « médias d'information » pour désigner les organisations médiatiques commerciales ou publiques composées notamment de journalistes professionnels, à savoir les chaînes de télévision publiques et privées, les quotidiens et hebdomadaires nationaux ou locaux, les stations radio et tous les sites Internet qui sont la propriété des catégories susnommées. Nous employons ces termes pour désigner ce qu'on appelle en anglais les «legacy media ». 
toujours de la presse traditionnelle, de la radio et de la télévision, (3) contrairement à l'actualité nationale, les «non-élites » sont entendues, ainsi que les voix discordantes ou marginales, (4) les actualités locales ne contiennent pas assez d'informations politiques et d'analyses approfondies, (5) l'émergence de médias alternatifs offre des opportunités pour de nouvelles relations et pratiques complémentaires aux médias traditionnels (2016, pp. 206 -227). Dans son étude sur la controverse entourant l'expulsion de propriétaires locaux à Philadelphie, Anderson (2010) a mis en évidence la multiplicité des acteurs participant à la production d'informations, à savoir principalement des médias traditionnels, des militants, des hommes politiques et des blogueurs. Dans ce cas particulier, la diffusion de l'information a été en partie organisée par des activistes, qui ont mobilisé des alliés politiques et des techniques journalistiques pour faire entendre leur voix. Dans une étude de l'écosystème de l'information de Baltimore, le Pew Research Center a constaté que, même s'il existe divers producteurs (sites web de chaînes de télévision locales, journaux traditionnels et spécialisés, blogs locaux), les médias traditionnels sont toujours garants de la couverture médiatique. Au cours des six événements analysés au cours d'une semaine type, les médias alternatifs ont produit peu de reportages et de matériaux originaux. De plus, les plateformes socionumériques habituelles ont été utilisées, mais principalement pour relayer des informations déjà produites sur un site web au public plus large (Pew Research Center, 2010). À cet égard, et en comparant les écosystèmes de Leeds (Royaume-Uni) et de Philadelphie (États-Unis), Anderson, Coleman et Thumim sont parvenus à la conclusion que les conversations interpersonnelles et les personnalités locales restent des relais importants, en marge des médias traditionnels dans les écosystèmes médiatiques locaux (2015). Toutes ces recherches montrent les reconfigurations des écosystèmes médiatiques locaux à l'époque contemporaine. Elles insistent sur la diversité des acteurs qui en font partie, ainsi que de leurs pratiques.

\subsection{La participation dans les études sur le journalisme : entre optimisme et pessimisme}

$\mathrm{Au}$ sein de ce que la recherche anglo-saxonne appelle les journalism studies, la notion de « journalisme participatif » fait aujourd'hui référence à une diversité de discours et de pratiques qui mettent en avant le rôle actif du public et des citoyens dans la production et la diffusion de contenus liés à l'actualité (Allan, 2013 ; Barnes, 2016 ; Bor- 
ger et al., 2013 ; Carpentier, 2015 ; Domingo et al., 2008 ; Paulussen et al., 2007 ; Wall, 2015). Le concept de journalisme participatif fait écho à des notions plus anciennes, telles que le «journalisme communautaire $^{3} »$ (Reader \& Hatcher, 2012) ou le « journalisme public » (Ahva, 2010).

La notion de journalisme participatif a gagné en popularité au cours de la dernière décennie, à mesure qu'avançait la numérisation, et ce tant pour les journalistes que pour les citoyens (Kreiss \& Brennen, 2016). La communauté des chercheurs s'est dès lors intéressée à plusieurs aspects du journalisme participatif, tels les caractéristiques des contenus générés (notamment leur crédibilité), l'opportunité économique de leur intégration ou, de manière plus générale, le rôle du public et les relations que celui-ci entretient avec les journalistes. Certaines recherches se sont intéressées au potentiel démocratique de la participation au journalisme (Borger et al., 2013). D'un côté, la participation au journalisme prend une connotation positive, est perçue comme vecteur de processus journalistiques démocratisés ainsi que d'un certain renouement entre les institutions journalistiques et leur public. D'un autre côté, cette participation est vue par certains chercheurs et professionnels comme problématique, la participation citoyenne étant parfois perçue comme incontrôlable ou non professionnelle, voire carrément biaisée (Quandt, 2018). Cette tension entre un idéal journalistique et un contrôle des professionnels d'une part, et cette culture des nouvelles technologies et de la participation d'autre part, est pour Lewis une tension clé qui tiraille les journalistes (Lewis, 2012). Anderson et Revers indiquent que cette vision positive de la participation n'est pas propre aux chercheurs, c'est également le sentiment d'une certaine « gauche politique ${ }^{4}$ », qui croit en la capacité des nouvelles technologies à permettre un journalisme plus horizontal, comme ce fut le cas pour le mouvement IndyMedia, presque à l'arrêt aujourd'hui (Anderson \& Revers, 2018). Cette vision de la participation est longtemps restée dominante dans les études sur le journalisme, car elle faisait écho non seulement à l'optimisme de certains journalistes professionnels, mais aussi à celui des managers et des chercheurs. Comme l'affirme Quandt: " pour le dire plus franchement, alors que la plupart des universitaires voulaient dire "sauver le journalisme" et "renforcer la démocratie", certains responsables des médias

3 Pour « community journalism ».

4 Anderson et Revers utilisent l'expression « political left» dans leur recherche. 
ont entendu "économiser de l'argent" et "renforcer notre entreprise" » (Quandt, 2018, p. 38).

Un deuxième type de discours scientifique sur la participation au journalisme est plus pessimiste. En effet, les recherches initiales sur la participation au journalisme se centraient sur des cas de participation « remarquables », « extrêmes » et peu révélateurs de la norme. Les données empiriques sur ce sujet provenaient essentiellement d'études de cas. Plus tard, des études quantitatives plus conséquentes ont montré le peu de motivation des utilisateurs à participer, ainsi que le scepticisme des journalistes par rapport à la capacité du public à créer des contenus de qualité (Quandt, 2018, pp. 36-40). Il n'a pas fallu attendre longtemps pour voir les données remettre en question les ambitions des professionnels et des chercheurs. Déjà, en 2008, Domingo et al. mentionnaient qu' « un examen plus approfondi séparant le processus de production des informations en cinq étapes a confirmé que seule l'étape de l'interprétation était sensiblement ouverte à une sorte de participation citoyenne dans les journaux étudiés. Le public n'a pas du tout participé à la sélection et au filtrage [des informations] » (Domingo et al., 2008, p. 34). Les fils de commentaires sur les sites de presse en ligne sont un exemple de cette remise en question : ils ont bien souvent disparu, sous-traités ou externalisés sur les réseaux sociaux (Anderson \& Revers, 2018 ; Quandt, 2018 ; Westlund \& Ekström, 2018). Par opposition aux visions positives de la participation, le concept de « participation obscure ${ }^{5}$ » s'est même développé pour désigner les campagnes de désinformation, les « fake news », la propagation des théories du complot et autres actes de trolling ${ }^{6}$ sur Internet (Quandt, 2018, pp. 40-43).

La reconfiguration des pratiques dans les écosystèmes médiatiques contemporains, ainsi que les résultats des recherches évoquées ci-dessus, nous poussent à nous écarter de l'analyse des dispositifs de « journalisme participatif » stricto sensu et nous invite à nous concentrer sur la participation au journalisme dans un sens plus large, en prenant en compte la diversité des pratiques et des acteurs qui interagissent durant la production et la diffusion de contenus liés à l'actualité. À ce titre, le journalisme local a attiré l'attention d'une communauté de chercheurs (Ahva \& Wiard, 2018, pp. 65-66). Souvent considéré comme une industrie en déclin (Nielsen, 2015), le journalisme local semble per-

5 Pour « dark participation ».

6 Entendu comme des actes volontairement destructeurs (violents, méchants, désobligeants, contre-productifs, etc.) de la part d'internautes. 
tinent à étudier, tant la participation du public au journalisme pourrait avoir un impact positif sur les organisations médiatiques. D'un point de vue méthodologique, la diversité linguistique et culturelle de Bruxelles, ainsi que la forte présence d'organisations médiatiques sur le territoire (Komorowski et al., 2018), en font un écosystème particulièrement fertile à analyser (Wiard, 2019; Wiard \& Pereira, 2018).

\section{Vers une grille d'analyse pour évaluer les pratiques participatives}

Une fraction des recherches liées à la participation au journalisme s'est attachée à l'évaluer en créant des modèles et des grilles d'analyse, dont la filiation peut être ramenée à « l'échelle de la participation citoyenne » d'Arnstein (1969). Un premier modèle divise la participation aux médias en cinq temps : l'accès/observation, la sélection/filtrage, le traitement/édition, la distribution et l'interprétation (Domingo et al., 2008 ; Singer et al., 2011). Ce modèle peut paraître aujourd'hui désuet, du fait que la production et la diffusion de contenus liés à l'actualité sur Internet et sur les réseaux sociaux ne sont pas linéaires. Néanmoins, il établit le fait que les pratiques participatives peuvent être diverses et se produire à différents moments de la production de contenus.

Le deuxième modèle existant est la typologie de la participation de l'audience de Kammer (2013). Dans une approche inductive proche de la théorie ancrée, l'auteur théorise les différents types de participation du public et les rôles des journalistes par rapport à celui-ci (Kammer, 2013, p. 118). Lors de cette recherche, il établit quatre idéaux types de la participation : l'information, la collaboration, la conversation, et la métacommunication (Kammer, 2013, pp. 119-122). La simplicité de ce modèle permet d'inclure une diversité de pratiques et de routines. De plus, il met, pour chaque type de participation, le rôle du public en regard de celui des journalistes professionnels.

Un troisième modèle classifie les pratiques de participation au journalisme en cinq orientations : la participation à travers le («through »), au («in ), avec le («with»), autour du («around ») et pour le («for») journalisme (Ahva, 2017, pp. 153-154). Dans le cadre de cette recherche saluée par la communauté scientifique ${ }^{7}$, Ahva avance l'utilité d'étudier la relation entre journalistes professionnels et public

7 L'article «How Is Participation Practiced by "In-Betweeners" of Journalism? » (2017) a notamment reçu le Bob Franklin Journal Article Award pour l'année 2018. 
comme dans les modèles précédents, mais inclut également une série d'autres acteurs, qu'elle nomme les « intermédiaires du journalisme ${ }^{8}$ » et qu'elle définit comme des citoyens qui ne sont pas des journalistes professionnels, mais qui sont néanmoins impliqués dans la production de contenus : activistes et personnes issues du milieu associatif, travailleurs indépendants, semi-professionnels, personnes issues du monde de la recherche, artistes locaux ou étudiants (2017, pp. 142-148).

La contribution la plus conséquente, en matière de classification des pratiques de participation, est sans conteste le modèle AIP (pour accès, interaction, participation) (Carpentier, 2011, 2012, 2015, 2016). Tout comme Domingo et al. (2008), Carpentier utilise la notion d'accès pour faire référence aux moyens de production et de distribution des contenus. Celle d'interaction fait, elle, référence à une forme d' « influence réciproque » (Carpentier, 2016, p. 51). La participation, enfin, sous-tend un équilibre entre les professionnels et d'autres catégories d'individus dans le processus de prise de décisions. C'est le cas, par exemple, des médias communautaires et alternatifs, au sein desquels des publics hétérogènes peuvent s'impliquer de diverses manières, non seulement dans la production et la circulation des contenus, mais également dans la gestion de l'organisation ou du projet (Carpentier, 2016, pp. 56-61). Ces composantes sont ensuite croisées avec quatre « domaines », à savoir la technologie, le contenu, les individus et les organisations (Carpentier, 2015).

Le modèle ADD (pour accès, dialogue, délibération) a été développé à la fin des années 2000 (voir à ce sujet Heikkilä \& Kunelius, 1998). Sur la base des recherches susmentionnées (Ahva, Heikkilä \& Kunelius, 2015 ; Ahva \& Wiard, 2018), il a été actualisé à deux reprises, avec l'objectif d'en faire une grille d'analyse opérationnelle des pratiques participatives au niveau local. Pour ce faire, les trois étapes de la participation (vue cette fois comme un continuum) ont été appliquées à trois domaines : la communauté de pratiques, les processus de production de contenu, et l'espace public ${ }^{9}$. Comme pour le modèle AIP (Carpentier, 2016), le modèle ADD divise la participation en trois dimensions, à savoir une dimension présentielle d'accès, une dimension plus dialogique et une dimension délibérative.

8 Pour « in-betweeners » en anglais.

9 L'espace public est ici à considérer en son sens commun et non au sens « habermacien » du terme. 
La première étape de la participation est celle de l'accès. Elle repose sur l'idée qu'il faut faire attention aux citoyens et leur reconnaître une place dans le processus de production des contenus d'information et qu'il est possible d'évaluer à quel point les portes du journalisme sont ouvertes au citoyen. À titre d'exemple, on peut citer l'accès à un studio de radio pendant une émission ou l'opportunité d'interpeller un journaliste présent à un événement (Ahva \& Wiard, 2018, p. 67). La deuxième étape du modèle est celle du dialogue. Une fois l'accès établi, un dialogue entre individus et producteurs d'informations peut potentiellement débuter. Dans le contexte de la production d'informations à l'échelle locale, cette pratique peut être comprise comme l'inclusion des citoyens dans une consultation extensive, ou un processus de réciprocité comme des contenus qui émergent de discussions (Heikkilä et Kunelius, 1998 ; Ahva et al., 2015 ; Ahva \& Wiard, 2018, p. 67). Enfin, l'étape de la délibération fait écho à la possibilité de codécision et donc à ce que Carpentier nomme la «participation » (Carpentier, 2016). L'étape de délibération pose la question de l'efficacité de la participation citoyenne et si celle-ci peut faire la différence. Au niveau des processus de production des contenus, les citoyens peuvent s'impliquer et ainsi décider de la forme finale de contenus et indirectement à la définition de ce qu'est l'actualité (Ahva \& Wiard, 2018, pp. 67-68).

Ce modèle est intéressant, car il a pour objectif d'analyser des projets qui vont au-delà du cadre du journalisme traditionnel. De plus, il dépasse certains paradoxes des modèles antérieurs, comme la dichotomie entre production et réception, qui ne semblent plus pertinents dans les écosystèmes médiatiques actuels (Carpentier, 2016 ; Kammer, 2013). Au vu des résultats des recherches sur les écosystèmes médiatiques locaux, il apparaît pertinent d'actualiser ce modèle en l'ouvrant à la diversité des catégories d'acteurs en présence à savoir les journalistes professionnels mais aussi les politiciens, les activistes, ou les citoyens. Le nombre de catégorie d'acteurs dépendra bien évidemment du contexte lors d'une application potentielle sur un terrain spécifique.

Les modèles évoqués ci-dessus s'inspirent bien évidemment les uns des autres, de la littérature sur la question ainsi que d'une série d'expériences empiriques de terrain. L'objectif de cette recherche est d'analyser la diversité des pratiques liées à la production de contenus liés à l'actualité locale à Bruxelles. Sur la base des précédentes recherches, nous proposons donc ici le modèle «ADD élargi » (figure 1), qui se centre sur les pratiques liées aux processus de production de l'information, tout en gardant la communauté de pratiques et l'espace public 
comme des données contextuelles. Ce modèle permet également d'envisager l'implication potentielle de diverses catégories d'acteurs.

\begin{tabular}{|l|l|l|l|}
\hline $\begin{array}{l}\text { Pratiques participatives/ } \\
\text { Types d'acteurs }\end{array}$ & Accès & Dialogue & Délibération \\
\cline { 1 - 1 } Catégorie d'acteurs 1 & \multirow{2}{*}{$\begin{array}{l}\text { Pratiques des différents acteurs } \\
\text { - en termes d'accès, de dialogue } \\
\text { et de délibération - leur permet- } \\
\text { tant de participer aux processus } \\
\text { de production de contenus. }\end{array}$} \\
\cline { 1 - 1 } Catégorie d'acteurs 2
\end{tabular}

Figure 1. Le modèle ADD élargi et centré sur la production de contenus liés à l'information

\section{Conclusion : du « journalisme participatif » à la « participa- tion au journalisme "}

L'utilisation du modèle «ADD élargi » pourrait apporter un éclairage nouveau sur les pratiques des acteurs participant à des écosystèmes médiatiques locaux. Loin des discours utopistes et technodéterministes argumentant qu'Internet va permettre le développement de nouveaux dispositifs participatifs en ligne, le modèle prend en compte la potentielle diversité des individus qui entrent en contact avec le journalisme hors ligne, et ce de façons variées. En effet, la littérature montre bien que les dispositifs dits de «journalisme participatif» en ligne sont rares et peu utilisés. Elle montre aussi que la participation se limite souvent à des formes au niveau de l'accès et de dialogue, et non à des formes maximalistes comprenant la participation aux prises de décisions sur la forme du contenu final. La contribution théorique de cet article réside dans la proposition de décentrer l'analyse de la participation aux médias, en se concentrant non pas sur une vision stricte du «journalisme participatif», mais sur une vision élargie de celui-ci, afin d'étudier la participation au journalisme dans sa globalité, c'està-dire la multiplicité des manières dont les stakeholders (individus et collectifs) prennent part à la production de contenus liés à l'actualité. Ainsi, cette étude propose modestement un modèle qui pourrait faire le lien entre les recherches empiriques sur le journalisme participatif au niveau local et celles portant sur la diversité des individus participant à l'espace public. Des études ultérieures pourraient affiner notre compré- 
hension de ces processus, notamment en comparant différents cas ou différents écosystèmes.

\section{Bibliographie}

Ahva, L. (2010). Making News with Ccitizens. Public Journalism and Professional Reflexivity in Finnish Newspapers. Tampere : Tampere University Press.

Ahva, L. (2017). How Is Participation Practiced by "In-Betweeners" of Journalism? Journalism Practice, 11(2-3), 142-159. Doi : https://doi.org/10.1080/17512786.20 16.1209084

Ahva, L., Heikkilä, H. \& Kunelius, R. (2015). Civic Participation and the Vocabularies for Democratic Journalism. The Routledge Companion to Alternative and Community Media, 155.

Ahva, L. \& Wiard, V. (2018). Participation in Local Journalism: Assessing Two Approaches through Access, Dialogue and Deliberation. Sur le journalisme. About Journalism. Sobre jornalismo, 7(2), 64-76. Disponible à : http://www.surlejournalisme.kinghost.net/rev/index.php/slj/article/view/359

Aldridge, M. (2007). Understanding the Local Media. McGraw-Hill Education (UK).

Allan, S. (2013). Citizen Witnessing: Revisioning Journalism in Times of Crisis. John Wiley \& Sons.

Anderson, C. W. (2010). Journalistic Networks and the Diffusion of Local News: The Brief, Happy News Life of the "Francisville Four". Political Communication, 27(3), 289-309. Doi : https://doi.org/10.1080/10584609.2010.496710

Anderson, C. W. (2016). News Ecosystems. Dans C. Anderson, T. Witschge, D. Domingo \& A. Hermida (eds.), The SAGE Handbook of Digital Journalism (pp. 410-423). Los Angeles, Sage. Disponible à : https://uk.sagepub.com/sites/default/files/upm-assets/75406_book_item_75406.pdf

Anderson, Chris W. (2013). Rebuilding the News. Metropolitan Journalism in the Digital Age, Temple University Press.

Anderson, C. W., Coleman, S. \& Thumim, N. (2015). How News Travels: A Comparative Study of Local Media Ecosystems in Leeds (UK) and Philadelphia (US). Dans R. K. Nielsen (ed.), Local Journalism: The Decline of Newspapers and the Rise of Digital Media (73-93). Londres, Bloomsbury/I.B. Tauris.

Anderson, C. W. \& Revers, M. (2018). From Counter-power to Counter-Pepe: The Vagaries of Participatory Epistemology in a Digital Age. Media and Communication, 6(4), 24-25. Doi : https://doi.org/10.17645/mac.v6i4.1492

Arnstein, S. R. (1969). A Ladder Of Citizen Participation. Journal of the American Institute of Planners, 35(4), 216-224. Doi : https://doi.org/10.1080/01944366908977225

Barnes, R. (2016). The Ecology of Participation. Dans C. Anderson, T. Witschge, D. Domingo \& A. Hermida (eds.), The SAGE Handbook of Digital Journalism (pp. 179-191). Los Angeles, Sage. Doi : http://dx.doi.org/10.4135/9781473957909. n12

Borger, M., van Hoof, A., Costera Meijer, I. \& Sanders, J. (2013). Constructing Participatory Journalism as a Scholarly Object: A Genealogical Analysis. Digital Journalism, 1(1), 117-134. https://doi.org/10.1080/21670811.2012.740267 
Bousquet, F. \& Smyrnaios, N. (2012). Les médias et la société locale, une construction partagée. Sciences de la société, 84-85, 5-15. Doi : https://doi.org/10.4000/sds. 1792

Bowman, S., \& Willis, C. (2003). We media. How audiences are shaping the future of news and information. Reston, Va, The Media Center at the American Press Institute. Disponible à : https://www.hypergene.net/wemedia/download/we_media.pdf

Carpentier, N. (2011). Media and Participation: A Site of Ideological-democratic Struggle. Bristol/Chicago: Intellect Books.

Carpentier, N. (2012). The Concept of Participation. If They Have Access and Interact, Do They Really Participate? Revista Fronteiras, 14(2). Doi : https://doi.org/10.4013/ fem.2012.142.10

Carpentier, N. (2015). Differentiating Between Access, Interaction and Participation. Conjunctions. Transdisciplinary Journal of Cultural Participation, 2(2), 7-28. Doi : https://doi.org/10.7146/tjcp.v2i2.23117

Carpentier, N. (2016). Différencier accès, interaction et participation. Dans P. Morelli, N. Pignard-Cheynel \& D. Baltazart (eds), Publics et TIC: Confrontations conceptuelles et recherches empiriques (pp. 45-69). Nancy : Presses universitaires de Nancy. Disponible à : http://www.diva-portal.org/smash/record.jsf?pid=diva2:889584

Coleman, S., Thumim, N., Birchall, C., Firmstone, J., Moss, G., Parry, K., Stamper, J. \& Blumler, J. G. (2016). The Mediated City: The News in a Post-Industrial Context. Londres : Zed Books Ltd.

Domingo, D., Quandt, T., Heinonen, A., Paulussen, S., Singer, J. B. \& Vujnovic, M. (2008). Participatory Journalism Practices in the Media and Beyond. Journalism Practice, 2(3), 326-342. https://doi.org/10.1080/17512780802281065

Domingo, D. \& Le Cam, F. (2014). Narrating Multiculturalism: Polyphonic News Discourses in Brussels. Paper session presented at Local Journalism around the World: Professional Practices, Economic Foundations, and Political Implications (26-28 février 2014: Oxford, Reuters Institute for the Study of Journalism.

Domingo, D., Masip, P. \& Costera Meijer, I. (2015). Tracing Digital News Networks: Towards an Integrated Framework of the Dynamics of News Production, Circulation and Use. Digital Journalism, 3(1), 53-67. Doi : https://doi.org/10.1080/21670 811.2014.927996

Franklin, B. (Ed.). (2005). Local Journalism and Local Media: Making the Local News. Londres: Routledge.

Heikkilä, H. \& Kunelius, R. (1998). Access, Dialogue, Deliberation. Experimenting with Three Concepts of Journalism Criticism. Nordicom REview, 19(1), 71-84. Disponible à : http://norden.diva-portal.org/smash/get/diva2:1534596/FULLTEXT01. pdf

Kammer, A. (2013). Audience Participation in the Production of Online News: Towards a Typology. NORDICOM Review: Nordic Research on Media and Communication, 34, 113-126. Doi : https://doi.org/10.2478/nor-2013-0108 Disponible à : https://www.nordicom.gu.se/sites/default/files/kapitel-pdf/09_kammer_0.pdf

Komorowski, M., Wiard, V., Derinöz, S., Picone, I., Domingo, D. \& Patriarche, G. (2018). Un médiapark à Bruxelles? L'industrie des médias et sa dynamique régionale. Brussels Studies: Notes de Synthèse, 129. Doi : https://doi.org/10.4000/brussels. 2058 
Kreiss, D. \& Brennen, J. S. (2016). Normative Theories of Digital Journalism. Dans C. W. Anderson, D. Domingo, A. Hermida, \& T. Witschge (Eds.), The SAGE Handbook of Digital Journalism (pp. 299-314). Los Angeles, Sage.

Le Cam, F. (2006). L'identité du groupe des journalistes du Québec au défi d'Internet. Université Rennes 1.

Le Cam, F. \& Domingo, D. (2015). The Plurality of Journalistic Identities in Local Controversies. Dans R. K. Nielsen (Éd.), Local Journalism: The Decline of Newspapers and Rise of Digital Media (pp. 99-116). Londres, Bloomsbury/I.B. Tauris.

Lewis, S. C. (2012). The Tension between Professional Control and Open Participation: Journalism and its Boundaries. Information, Communication \& Society, 15(6), 836866. Doi : https://doi.org/10.1080/1369118X.2012.674150

Lowrey, W. (2012). Journalism Innovation and the Ecology of News Production: Institutional Tendencies. Journalism \& Communication Monographs, 14(4), 214-287. https://doi.org/10.1177/1522637912463207

Napoli, P. M., Stonbely, S., McCollough, K. \& Renningen, B. (2015). Assessing the Health of Local Journalism Ecosystems. New Brunswick: Rutgers University. Disponible à : http://wp.comminfo.rutgers.edu/mpii-new/wp-content/uploads/ sites/129/2015/06/Assessing-Local-Journalism_Final-Draft-6.23.15.pdf.

Nielsen, R. K. (2015). Introduction: The Uncertain Future of Local Journalism. Dans R. K. Nielsen (Éd.), Local Journalism: The Decline of Newspapers and Rise of Digital Media (pp. 1-26). Londres, Bloomsbury/I.B. Tauris.

Paulussen, S., Heinonen, A., Domingo, D. \& Quandt, T. (2007). Doing It Together: Citizen Participation In The Professional News Making Process. Observatorio (OBS*) Journal, 3, 131-154. Doi : https://doi.org/10.15847/obsOBS132007148

Pélissier, N. \& Diouma Diallo, M. (2015). Le journalisme à l'épreuve des dispositifs socionumériques d'information et de communication. Revue française des sciences de l'information et de la communication, 6, 111. Doi : https://doi.org/10.4000/rfsic. 1449

Pew Research Cenrer. (2010). How News Happens. A Study of the News Ecosystem of One American City. Pew Research Center's Journalism Project. Disponible à : http://www.journalism.org/2010/01/11/how-news-happens/

Powers, M., Vera Zambrano, S., \& Baisnée, O. (2015). The News Crisis Compared: The Impact of the Journalism Crisis on Local News Ecosystems in Toulouse (France) and Seattle (US).Dans R. K. Nielsen (Éd.), Local Journalism: The Decline of Newspapers and Rise of Digital Media (pp. 31-50). Londres, Bloomsbury/I.B. Tauris.

Quandt, T. (2018). Dark Participation. Media and Communication, 6(4), 36-48. Doi : https://doi.org/10.17645/mac.v6i4.1519

Reader, B. \& Hatcher, J. A. (2012). Foundations of Community Journalism. Thousand Oaks: Sage.

Reese, S. D., Rutigliano, L., Hyun, K., \& Jeong, J. (2007). Mapping the Blogosphere: Professional and Citizen-based Media in the Global News Arena. Journalism, 8(3), 235-261. Doi : https://doi.org/10.1177/1464884907076459

Ryfe, D., Mensing, D., Ceker, H. \& Gunes, M. (2012). Popularity is not the Same Thing as Influence: A Study of the Bay Area News System. Journal of the International Symposium of Online Journalism (\# ISOJ), 2. Disponible à : https://isoj.org/wpcontent/uploads/2018/01/Mensing.pdf 
Singer, J. B., Domingo, D., Heinonen, A., Hermida, A., Paulussen, S., Quandt, T., Reich, Z. \& Vujnovic, M. (2011). Participatory Journalism: Guarding Open Gates at Online Newspapers. John Wiley \& Sons.

Wahl-Jorgensen, K. (2016). The Chicago School and Ecology: A Reappraisal for the Digital Era. American Behavioral Scientist, 60(1), 8-23. Doi : https://doi. org/10.1177/0002764215601709

Wall, M. (2015). Citizen Journalism: A Retrospective on What we Know, an Agenda for What we Don't. Digital Journalism, 3(6), 797-813. Doi : https://doi.org/10.108 0/21670811.2014.1002513

Westlund, O. \& Ekström, M. (2018). News and Participation through and beyond Proprietary Platforms in an Age of Social Media. Media and Communication, 6(4), 1-10. Doi : ttps://doi.org/10.17645/mac.v6i4.1775

Wiard, V. (2019). News Ecology and News Ecosystems. Oxford Research Encyclopedia of Communication.

Wiard, V. \& Pereira, F. H. (2018). Bad Neighborhoods in a Good City? Space, Place and Brussels's Online News. Journalism Studies, 20(5), 649-674. Doi : https://doi. org/10.1080/1461670X.2017.1417052

\section{@) $\Theta \Theta$}

«Attibution - pas d'utilisation Commerciale - Pas de Modification 4.0. International» (CC BY-NC-ND) 